\title{
Resource Allocation for User-Relay Assisted MISO-OFDMA Networks
}

\author{
İlhan BAŞTÜRK \\ Electrical and Electronics Engineering Department, \\ Adnan Menderes University, Aydın, Turkey \\ Email: ilhan.basturk@adu.edu.tr
}

\author{
Berna ÖZBEK \\ Electrical and Electronics Engineering Department, \\ Izmir Institute of Technology, Izmir, Turkey \\ Email: bernaozbek@iyte.edu.tr
}

\begin{abstract}
The next generation cellular networks request higher capacity and coverage that user-relay assisted Orthogonal Frequency Division Multiple Access (OFDMA) networks are costeffective solutions to meet these demands. These networks can be enriched with multiple antennas in order to obtain a diversity gain to combat signal fading and to achieve more capacity gain without increasing the bandwidth or transmit power. However, radio resource management $(\mathrm{RRM})$ such as relay selection and resource allocation $(\mathrm{RA})$ is important in such a multi-user, multirelay and multi-antenna environment to fully take advantage of multiple-input single-output (MISO)-OFDMA based user-relay assisted cellular networks. Thus, we propose a RA algorithm for these networks to reveal the benefits of the combination of userrelaying and multiple antennas technologies and compare the proposed scheme with existing schemes in terms of percentage of satisfied users and cell-edge users' data rate.
\end{abstract}

\section{INTRODUCTION}

OFDMA is a good candidate to meet the ubiquitous high data rate coverage expectation of the next-generation wireless communication networks because of its inherent robustness against frequency-selective fading, high spectral efficiency and flexible resource allocation. Multiple-input multiple-output (MIMO) technology, that uses multi antenna arrays either on the transmitter or receiver side, also offers significant increase in the data rate without additional bandwidth or transmit power. Using OFDMA with MIMO gives rise to greater system capacity and improves the reliability. On the other hand, relaying technology has attracted significant attention of many researchers, due to its potential benefits of enlarging the coverage and increasing the capacity. The combination of MIMO-OFDMA and relaying is one of the key technologies to deliver the promise of the reliable and high-data-rate coverage in the most cost effective manner.

Relay assisted networks can be examined as fixed and mobile relay networks according to relay movement [1][2]. The relay stations (RSs) are part of the network infrastructure in fixed relay networks but not in mobile relay networks. Fixed relay networks have been extensively studied in the literature, and have already been included in the 4G Long Term Evolution (LTE)-Advanced standard [3]. In mobile relay networks, the mobile RSs whose locations are not deterministic can be examined in two different forms such as dedicated mobile RSs that are mounted on moving vehicles or user terminals acting as mobile RSs which are also called as userrelays. While fixed relaying brings improvements in cellular

978-1-4673-6540-6/15/\$31.00 (c) 2015 IEEE systems, the implementation of user-relaying will bring a huge gain to $5 \mathrm{G}$ based wireless networks with employing different scenarios. Thus, user-relaying is foreseen as one of the emerging technologies that will change and define the next generation telecommunication standard [4][5].

In OFDMA-based user-relay assisted networks, capacity and coverage benefits can be revealed by developing efficient RRM algorithms such as relay selection and RA. The RRM for user-relay assisted networks is examined for different optimization objectives in [6]-[12]. In [6], power minimization problem has been handled and optimal and sub-optimal solutions have been presented for amplify and forward (AF) relaying scheme. In [7], the fairness issue has been considered and subcarrier and power allocation schemes have been examined for uplink OFDMA. In [8], the joint optimization of relay selection, relay-strategy selection such as $\mathrm{AF}$ or decode and forward (DF) and RA have been studied in order to maximize an utility function for cellular networks. In [9], an optimal cooperation strategy has been developed for two-user uplink OFDMA systems. In [8] and [9], it has been assumed that nodes are able to transmit and receive simultaneously on adjacent subcarriers and the feasibility of this assumption has been examined in [10]. We have proposed sub-optimal practical RRM solutions for the user-relay assisted OFDMA networks in [11][12]. In [11], heterogeneous networks which contain macro base stations (BSs) and access points have been combined with the user-relaying technology and efficient relay selection, network interface selection and RA algorithms have been presented for such a complex environment. In [12], a joint relay selection and RA algorithm has been examined by considering the queue information of each user. All these relay selection and RA algorithms for user-relay assisted cellular networks are applied to single-input single-output (SISO)OFDMA systems.

In this paper, we combine OFDMA based multiple antennas technology with user-relaying. We propose RA algorithms for MISO-OFDMA based user-relay assisted networks since the algorithms presented for SISO-OFDMA systems can not be directly applied to the MISO-OFDMA systems in which more than one user is supported per subchannel. The proposed user-relay assisted MISO-OFDMA scheme is compared to non-relaying MISO-OFDMA and fixed-relay assisted MISOOFDMA schemes and also user-relay assisted SISO-OFDMA scheme.

The rest of this paper is organized as follows. In Section II, we give the system model. In Section III, we present the 
proposed RA algorithm for user-relay assisted MISO-OFDMA networks. In Section IV, performance evaluations are examined and finally, the conclusions are given in Section V.

\section{SYSTEM MODEL}

In this paper, single cell downlink MISO-OFDMA based user-relay assisted network topology is used as illustrated in Figure 1. The BS that has $N_{t}$ transmit antennas is located at the cell center and $K$ users with single receive antennas are deployed randomly in the cell whose radius is $R$. The cell area is partitioned as inner $(0-(2 R / 3))$ and outer $((2 R / 3)-R)$ areas. $M$ and $L$ users are deployed in the inner and outer areas, respectively. $M$ inner users are permitted to communicate to the BS directly and $L$ outer or cell-edge users are allowed to communicate to the BS either directly or via another user called as user-relay. Inner users are not only the users but also the user-relay candidates of the cell-edge users. The cell edge users' relay candidates can be determined by using the area whose radius is $R / 2$ as shown in Figure 1 in order to decrease the computational complexity. The DF relaying strategy and two-hop relaying is considered in this study.

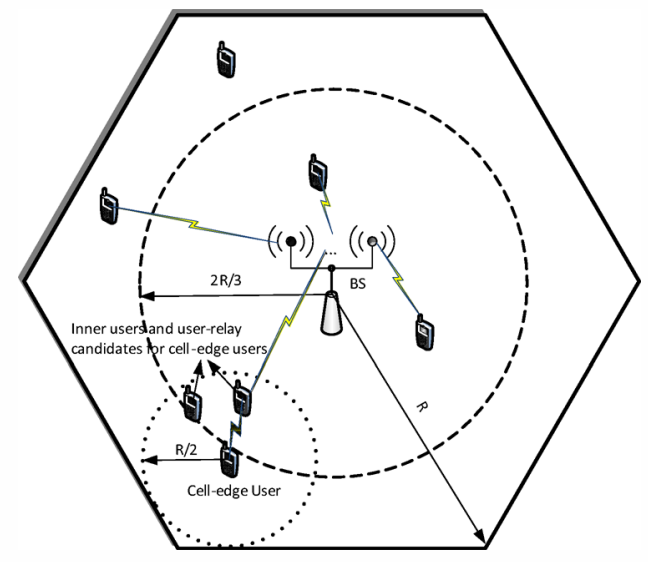

Fig. 1: User-relay assisted MISO-OFDMA network topology.

A generic frame structure in which the downlink frame is divided into two consecutive subframes, BS subframe followed by RS subframe is used. The subframe durations are selected equal in this study. In the first subframe called BS subframe, $\mathrm{BS}$ transmits data to mobile stations (MSs) and RSs (BS $\rightarrow$ MS and $\mathrm{BS} \rightarrow \mathrm{RS}$ ) and in the second subframe called RS subframe, MSs receive data from $\mathrm{BS}$ and $\mathrm{RSs}(\mathrm{BS} \rightarrow \mathrm{MS}$ and $\mathrm{RS} \rightarrow \mathrm{MS}$ ).

In our model, the BS has $N_{t}$ transmit antennas so it can transmit to multiple nodes simultaneously at each subchannel. As in the practical scenarios, total number of nodes that BS will serve is larger than the number of transmit antennas, $K+M>N_{t}$. Thus, it is required to select $a \leq N_{t}$ links for each subchannel. The number of simultaneously served links on each subchannel is limited by the number of transmit antennas. There are, $\Theta=\sum_{a=1}^{N_{t}}\left(\begin{array}{c}K+M \\ a\end{array}\right)$ possible combinations of links in the same subchannel. The scheduled users' and/or user-relays' set on subchannel $n$ is denoted as $\mathbb{X}_{\pi, n}$ whose cardinality is given as $0<\left|\mathbb{X}_{\pi, n}\right| \leq N_{t}$ where $\pi=1,2, \ldots, \Theta$. Let assume a set of users and/or user-relays,
$\mathbb{X}_{\pi, n}=\left\{x_{1}, x_{2}, \cdots, x_{a}\right\}$, that produces the channel matrix which consists of $a$ channel vectors belonging to these selected nodes for subchannel $n$;

$$
\mathbf{H}_{n}\left(\mathbb{X}_{\pi, n}\right)=\left[\mathbf{h}_{0, x_{1}, n}^{T} \cdots \mathbf{h}_{0, x_{\bullet}, n}^{T}\right]^{T}
$$

where $\mathbf{h}_{0, x, n}$ is $1 \times N_{t}$ channel vector between the BS and node $x_{a}$.

When multiple nodes are served simultaneously on the same subchannel, it causes interference among the nodes. In order to overcome this problem, Zero-Forcing beamforming (ZF-BF) technique is utilized. The ZF-BF matrix on subchannel $n$ is calculated as follows,

$$
\mathbf{W}_{n}\left(\mathbb{X}_{\pi, n}\right)=\lambda\left(\mathbf{H}_{n}\left(\mathbb{X}_{\pi, n}\right)\right)^{H}\left[\left(\mathbf{H}_{n}\left(\mathbb{X}_{\pi, n}\right)\right)\left(\mathbf{H}_{n}\left(\mathbb{X}_{\pi, n}\right)\right)^{H}\right]^{-1}
$$

where $\mathbf{W}_{n}\left(\mathbb{X}_{\pi, n}\right)=\left[\mathbf{w}_{0, x_{1}, n} \cdots \mathbf{w}_{0, x_{\bullet}, n}\right]$ that contains $N_{t} \times 1$ beamforming vectors belonging to each node on subchannel $n$ and $\lambda$ is calculated as,

$$
\lambda=\frac{1}{\sqrt{\left.\operatorname{tr}\left[\left(\left(\mathbf{H}_{n}\left(\mathbb{X}_{\pi, n}\right)\right) \mathbf{H}_{n}\left(\mathbb{X}_{\pi, n}\right)\right)^{H}\right)^{-1}\right]}}
$$

The data rate between the BS and any node (user or userrelay) $j \in \mathbb{X}_{\pi, n}$ on subchannel $n$ is calculated as;

$$
R_{0, j, n, \pi}=\frac{B}{N} \log _{2}\left(1+\gamma_{0, j, n, \pi}\right)
$$

where $\gamma_{0, j, n, \pi}=\frac{P_{0, j, n, \pi}\left|\mathbf{h}_{0, j, n} \mathbf{w}_{0, j, n}\right|^{2}}{N_{0}(B / N)}$ is the Signal to Noise Ratio (SNR) value between the BS and receiver node $j$ on subchannel $n . P_{0, j, n, \pi}$ is the transmitted power per subchannel per node, $\mathbf{h}_{0, j, n}$ is the channel coefficient vector between BS and node $j$ that includes pathloss, shadowing and multipath, $B$ is the available bandwidth and $N_{0}$ is the noise power density.

If a cell-edge user $c$ communicates to the BS via user-relay node $m \in \mathbb{M}$ where $\mathbb{M}=\{1,2, \ldots, M\}$ is the inner users' set, the data rate between user-relay and user is calculated as,

$$
R_{m, c, n}=\frac{B}{N} \log _{2}\left(1+\gamma_{m, c, n}\right)
$$

where $\gamma_{m, c, n}=\frac{P_{m, c, n}\left|h_{m, c, n}\right|^{2}}{N_{0}(B / N)}$ is the SNR between a transmitter node $m$ and receiver node $c$ on subchannel $n$. $P_{m, c, n}$ is the transmitted power per subchannel, $h_{m, c, n}$ is the channel coefficient between two nodes $(m$ and $c$ ) that includes pathloss, shadowing and multipath.

\section{THE Proposed RA ALGORITHM FOR USER-RELAY ASSISTED MISO-OFDMA NETWORKS}

In this section, we propose a sub-optimal RA algorithm for user-relay assisted MISO-OFDMA networks to satisy user's data rate requirements while maximizing sum rate. The relay selection is performed at the BS by using Minimum Total Pathloss (MTP) criteria in [13], thus $r_{k}$, the relay candidate of user $k$ is known at the BS. The proposed algorithm has an Initialization step and two main steps as Step 1 and Step 2 . In the algorithm, we assume that the user-relays receive and transmit the data using the same subchannel in two consecutive 
subframes. If a subchannel in the first subframe is allocated to a relayed user that communicates to the $\mathrm{BS}$ with the help of a user-relay in Step1, the same subchannel in the second subframe will also be reserved because of the twohop relaying. Thus, this subchannel can not be used for RA in Step2.

In the first step, the subchannels are allocated to the direct users which communicate to the BS directly and relayed users. Multiple users can be allocated to each subchannel since the BS has $N_{t}$ transmit antennas. Only one of these users can be scheduled as user-relay because the RS $\rightarrow$ MS is a SISO link. The first node on subchannel $n$ is the best BS link that has the maximum rate value out of all potential links. New users and/or user-relays are added to subchannel $n$ in respect of the given conditions: additional node should be as much as orthogonal to the already selected nodes and it should increase the sum data rate of $n$. In order to determine an orthogonal user set for the already selected nodes, a projector matrix, $\mathbf{P}^{\perp}$ is calculated [14] and each remaining channel vectors belonging to users not selected yet are projected onto the orthogonal complement of the subspace spanned by the channels of the selected nodes. Thereafter, $\mathcal{T}^{(1)}$, whose value is decided heuristically as $\min \left\{|\mathbb{Z} \cup \mathbb{R}|, N_{t}\right\}$, orthogonal users and/or user-relays are selected to form a group, $\zeta$. Then, the best link that has the maximum rate value is selected through $\zeta$. If this link increases the sum rate of subchannel $n$, it is admitted. The final rate values of each user are updated after allocating all selected nodes on subchannel $n$. Each user has its own threshold data rate, $R_{k}^{t h}, \forall k$, and the user which reaches its threshold data rate is called as satisfied user and the user that does not reach this rate is called as unsatisfied user. This step is repeated until all subchannels are exhausted or all users are satisfied.

In the second step of the algorithm, the remaining subchannels in the second subframe are only allocated to the direct users based on their rate values as in Step1. In this step, the value of $\mathcal{T}^{(2)}$ is decided heuristically as $\min \left\{|\mathbb{Z}|, N_{t}\right\}$. This step is terminated when all users are satisfied or all subchannels are exhausted. The proposed RA algorithm is given in detail below. In the algorithm, the rate values are calculated by using Equations 4 and 5 but notice that index $\pi$ is omitted for the rate calculation of the Equation 4, because it refers to specific set of users.

\section{Proposed RA Algorithm}

Let $\mathbb{N}, \mathbb{N}^{\prime}$ be the set of subchannels at subframe one and two, respectively. $\mathbb{U}$ and $\mathbb{S}$ are the unsatisfied and satisfied users sets, respectively.

\section{- Initialization}

$\star \mathbb{U}=\{1,2, \ldots, K\}, \mathbb{S}=\{\emptyset\}, R_{k}=0, \forall k \in \mathbb{U}$

$\star \mathbb{N}=\mathbb{N}^{\prime}=\{1,2, \ldots, N\}, n=1$.

- Step1

while $\mathbb{U} \neq \emptyset$ and $n \leq N$ do

$*$ Set $\left|\mathbb{X}_{n}\right|=\emptyset, \mathbb{Z}=\mathbb{U}, \mathbb{R}=\mathbb{M}$ and $a=1$

* Determine the data rate $R_{k, n} ; \forall k \in \mathbb{Z}$

if the user-relay index $r_{k} \neq \emptyset$ do

$$
\begin{aligned}
R_{k, n} & =\max \left\{R_{0, k, n}, \min \left\{R_{0, r_{k}, n}, R_{r_{k}, k, n}\right\}\right\} \\
\text { else do } & \\
R_{k, n} & =R_{0, k, n}
\end{aligned}
$$$$
\text { end if }
$$

* Find user $k^{\prime}$ that satisfies, $k^{\prime}=\arg \max _{k \in \mathbb{Z}} R_{k, n}$

if user $k^{\prime}$ communicates BS through a user-relay do ○ $\mathbb{X}_{n}(a)=\left\{r_{k^{\prime}}\right\}$ where $\mathbb{X}_{n}(a)$ shows the allocation result of the step $a$ and $\mathbf{H}\left(\mathbb{X}_{n}(a)\right)=\mathbf{h}_{0, r}{ }_{k^{\prime}, n}$

$\circ$ Set $\mathbb{R}=\emptyset$ to avoid to add any relay to that subchannel.

$\circ$ Reserve subchannel $n$ in the second subframe for relaying, $\mathbb{N}^{\prime} \leftarrow \mathbb{N}^{\prime} \backslash n$.

○ If $r_{k^{\prime}} \in \mathbb{Z}$, then $\mathbb{Z} \leftarrow \mathbb{Z} \backslash\left\{r_{k^{\prime}}\right\}$.

else do

$$
\begin{aligned}
& \circ \mathbb{X}_{n}(a)=\left\{k^{\prime}\right\} \text { and } \mathbf{H}\left(\mathbb{X}_{n}(a)\right)=\mathbf{h}_{0, k^{\prime}, n} . \\
& \circ \mathbb{Z} \leftarrow \mathbb{Z} \backslash\left\{k^{\prime}\right\} . \text { If } k^{\prime} \in \mathbb{R} \text {, then } \mathbb{R} \leftarrow \mathbb{R} \backslash\left\{k^{\prime}\right\}
\end{aligned}
$$

end if

* Compute the achievable rate for subchannel $n, \mathcal{R}\left(\mathbb{X}_{n}(a)\right)$.

$$
\mathcal{R}\left(\mathbb{X}_{n}(a)\right)=\sum_{j \in \mathbb{X}_{n}(a)} b_{j} \text { where } b_{j}=R_{0, j, n}
$$

* Add new users and/or user-relays to subchannel $n$.

while $a \leq N_{t}$ do

$\star$ Increase a by 1 ,

$\star$ Find as much as orthogonal users to the already selected ones in $\mathbb{X}_{n}(a-1)$ by using the projector matrix, $\mathbf{P}_{a}^{\perp}$. . $\mathbf{P}_{a}^{\perp}=\mathbf{I}_{N_{t}}-\overline{\mathbf{H}}^{H}\left(\overline{\mathbf{H}}^{H} \overline{\mathbf{H}}^{H}\right)^{-1} \overline{\mathbf{H}}$, where $\overline{\mathbf{H}}=\mathbf{H}_{n}\left(\mathbb{X}_{n}(a-1)\right)$. $\cdot q_{\kappa}=\mathbf{h}_{0, \kappa, n} \mathbf{P}_{a}^{\perp} \mathbf{h}_{0, \kappa, n}^{H}, \forall \kappa \in \mathbb{Z} \cup \mathbb{R}$

- Select the largest valued $\mathcal{T}^{(1)}$ users and/or user-relays through $q_{\kappa}$ to form a set, $\zeta$.

$\star$ Calculate the rate values of each link $\alpha \in \zeta$.

if $\alpha \in \mathbb{R}$ and $\alpha \notin \mathbb{Z}$ do

$\alpha$ is user-relay of $c^{\alpha}$ and $R_{c^{\alpha}, n}=\min \left\{R_{0, \alpha, n}, R_{\alpha, c^{\alpha}, n}\right\}$ else do

$\alpha$ is direct user and $R_{\alpha, n}=R_{0, \alpha, n}$

end if

$\star$ Find the best link $\beta \in \zeta$ that has the maximum rate value.

$\star$ Decide admitting this link to subchannel $n$.

if $\mathcal{R}\left(\mathbb{X}_{n}(a-1) \cup\{\beta\}\right) \geq \mathcal{R}\left(\mathbb{X}_{n}(a-1)\right)$ do $\circ \mathbb{X}_{n}(a)=\mathbb{X}_{n}(a-1) \cup\{\beta\}$,

○ $\mathcal{R}\left(\mathbb{X}_{n}(a)\right)=\mathcal{R}\left(\mathbb{X}_{n}(a-1) \cup\{\beta\}\right)$

$\circ \mathbf{H}\left(\mathbb{X}_{n}(a)\right)=\left[\mathbf{H}\left(\mathbb{X}_{n}(a-1)\right)^{T} \mathbf{h}_{0, \beta, n}^{T}\right]^{T}$.

○ If $\beta \in \mathbb{Z}, \mathbb{Z} \leftarrow \mathbb{Z} \backslash\{\beta\}$. If $\beta \in \mathbb{R}, \mathbb{R} \leftarrow \mathbb{R} \backslash\{\beta\}$

$\circ$ If $\beta$ is a user-relay,

Set $\mathbb{R}=\emptyset$ to avoid to add any relay to $n$.

Reserve subchannel $n$ in the second subframe for relaying $\mathbb{N}^{\prime} \leftarrow \mathbb{N}^{\prime} \backslash n$.

\section{else do}

o Terminate the allocation loop in $n$.

\section{end if}

\section{end while}

* Update the rate values of each link $j \in \mathbb{X}_{n}(a)$

if $j$ is a user-relay do

- BS serves $c^{j}$ over $j$.

- $R_{c^{j}, n}=\min \left\{R_{0, j, n}, R_{j, c^{j}, n}\right\}$ and $R_{c^{j}}=R_{c^{j}}+R_{c^{j}, n}$ else do If $R_{c^{j}} \geq R_{c^{j}}^{\text {th }}$ then $\mathbb{U} \leftarrow \mathbb{U} \backslash\left\{c^{j}\right\}, \mathbb{S} \leftarrow \mathbb{S} \cup\left\{c^{j}\right\}$.

- BS serves to direct user $j$.

end if

$R_{j, n}=R_{0, j, n}$ and $R_{j}=R_{j}+R_{j, n}$

If $R_{j} \geq R_{j}^{t h}$ then $\mathbb{U} \leftarrow \mathbb{U} \backslash\{j\}, \mathbb{S} \leftarrow \mathbb{S} \cup\{j\}$.

* Increase $n$ by 1 .

end while

- Step2

while $\mathbb{U} \neq \emptyset$ and $\mathbb{N}^{\prime} \neq \emptyset$ do

* Select the minimum indexed subchannel $n^{\prime}$ through the 
remaining subchannels of $\mathbb{N}^{\prime}$.

* Set $\left|\mathbb{X}_{n^{\prime}}\right|=\emptyset, \mathbb{Z}=\mathbb{U}$ and $a=1$

* Determine the data rates, $R_{k, n^{\prime}}=R_{0, k, n^{\prime}}, \forall k \in \mathbb{Z}$

* Find user $k^{*}$ that satisfies, $k^{*}=\arg \max _{k \in \mathbb{Z}} R_{k, n^{\prime}}$

$\circ \mathbb{X}_{n^{\prime}}(a)=\left\{k^{*}\right\}, \mathbb{Z} \leftarrow \mathbb{Z} \backslash\left\{k^{*}\right\}$ and $\mathbf{H}\left(\mathbb{X}_{n^{\prime}}(a)\right)=\mathbf{h}_{0, k^{*}, n^{\prime}}$

$*$ Compute the achievable rate for subchannel $n^{\prime}, \mathcal{R}\left(\mathbb{X}_{n^{\prime}}(a)\right)$.

* Add new users to subchannel $n^{\prime}$.

while $a \leq N_{t}$ do

$\star$ Increase a by 1 ,

$\star$ Find as much as orthogonal users to the already selected ones in $\mathbb{X}_{n^{\prime}}(a-1)$ by using the projector matrix, $\mathbf{P}_{a}^{\perp}$.

$\cdot q_{u}=\mathbf{h}_{0, u, n^{\prime}} \mathbf{P}_{a}^{\perp} \mathbf{h}_{0, u, n^{\prime}}^{H}, \forall u \in \mathbb{Z}$

- Select $\mathcal{T}^{(2)}$ users through $q_{u}$ to form $\Lambda$.

$\star$ Find the best link $z \in \Lambda$ that has the maximum rate value.

if $\mathcal{R}\left(\mathbb{X}_{n^{\prime}}(a-1) \cup\{z\}\right) \geq \mathcal{R}\left(\mathbb{X}_{n^{\prime}}(a-1)\right)$ do

$\circ \mathbb{X}_{n^{\prime}}(a)=\mathbb{X}_{n^{\prime}}(a-1) \cup\{z\}$ and $\mathbb{Z} \leftarrow \mathbb{Z} \backslash\{z\}$

$\circ \mathcal{R}\left(\mathbb{X}_{n^{\prime}}(a)\right)=\mathcal{R}\left(\mathbb{X}_{n^{\prime}}(a-1) \cup\{z\}\right)$

$\circ \mathbf{H}\left(\mathbb{X}_{n^{\prime}}(a)\right)=\left[\mathbf{H}\left(\mathbb{X}_{n^{\prime}}(a-1)\right)^{T} \mathbf{h}_{0, z, n}^{T}\right]^{T}$.

else do

○ Terminate the allocation loop in $n^{\prime}$.

end if

end while

* Update the rate values of each link $j \in \mathbb{X}_{n^{\prime}}(a)$

$$
\begin{aligned}
& \text { - } R_{j, n^{\prime}}=R_{0, j, n^{\prime}} \text { and } R_{j}=R_{j}+R_{j, n^{\prime}} \\
& \text {. If } R_{j} \geq R_{j}^{t h} \text { then } \mathbb{U} \leftarrow \mathbb{U} \backslash\{j\}, \mathbb{S} \leftarrow \mathbb{S} \cup\{j\} .
\end{aligned}
$$

$* \mathbb{N}^{\prime} \leftarrow \mathbb{N}^{\prime} \backslash n^{\prime}$

end while

\section{PERFORMANCE EVAluations}

In this section, the performance results are obtained to reveal the benefits of the proposed RA algorithm for userrelay assisted MISO-OFDMA networks in which the BS has $N_{t}=4$ transmit antennas and all users have $N_{r}=1$ receive antenna. The percentage of cell-edge users is selected as $10 \%$. The simulation results are examined for the case that the users have different threshold data rate values since the users can use different traffic types such as voice, video and data in practice. It is assumed that $40 \%$ of the users request $168 \mathrm{kbps}$, $30 \%$ of the users request $336 \mathrm{kbps}$ and $30 \%$ of the users request $504 \mathrm{kbps}$ in the cell area. In the simulations, we use the parameters as summarized in Table I.

TABLE I: Simulation Parameters

\begin{tabular}{|l|l|}
\hline Parameter & Value \\
\hline Frequency & $2 \mathrm{GHz}$ \\
\hline Bandwidth & $20 \mathrm{MHz}$ \\
\hline Thermal Noise Density & $-134.89 \mathrm{dBm} / \mathrm{Hz}$ \\
\hline$N_{t} \times N_{r}$ antennas & $4 \times 1$ \\
\hline BS Tx power & $46 \mathrm{dBm}$ \\
\hline Fixed relay Tx power & $37 \mathrm{dBm}$ \\
\hline User-relay Tx power & $23 \mathrm{dBm}$ \\
\hline Cell radius (R) & $500 \mathrm{~m}$ \\
\hline UE min. close-in distance to BS & $35 \mathrm{~m}$ \\
\hline Frame Duration & $10 \mathrm{~ms}$ \\
\hline Pathloss model & $\mathrm{BS} \rightarrow \mathrm{MS}, \mathrm{BS} \rightarrow \mathrm{RS}$ \\
& and RS $\rightarrow$ MS \\
& $128.1+37.6 \log _{1} \bullet d(\mathrm{~km})$ \\
\hline Shadowing model & Lognormal distribution, \\
& $\mu=0, \sigma=10 d B$ \\
\hline Multipath model & Extended Pedestrian A (EPA) \\
\hline
\end{tabular}

First of all, we compare the proposed user-relay assisted MISO-OFDMA scheme with two different MISO-OFDMA schemes. In the first scheme that is called $w / o$ relay, all users connect to the BS directly during the whole transmission frame so there is no relay in the system. In this scheme, the RA is performed by using a greedy algorithm that assigns the available resources to the users sequentially based on their achievable rate values until the users are satisfied. The second one is the fixed relay assisted scheme in which 3 fixed relays at equal angles and equal distances $(2 R / 3)$ to the BS are located. The path loss, shadowing and multipath channel parameters for all links except the $\mathrm{BS} \rightarrow \mathrm{RS}$ link is the same with user-relay assisted scheme and given in Table I. The BS $\rightarrow$ RS link has $4 d B$ lognormal shadowing and experience Rician fading with a Rician factor of $10 \mathrm{~dB}$. In this scheme, the relay selection in which MTP criteria [13] is used and RA in which the available resources are allocated to the users sequentially based on their achievable rate values until the users are satisfied are performed by the BS.

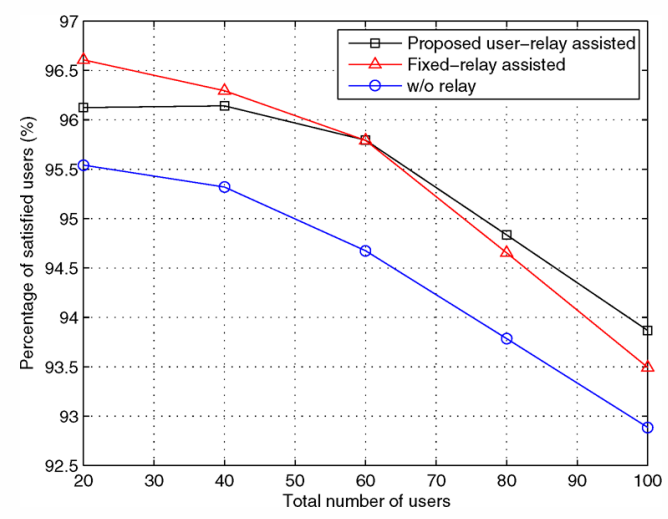

Fig. 2: Percentage of satisfied users for MISO-OFDMA

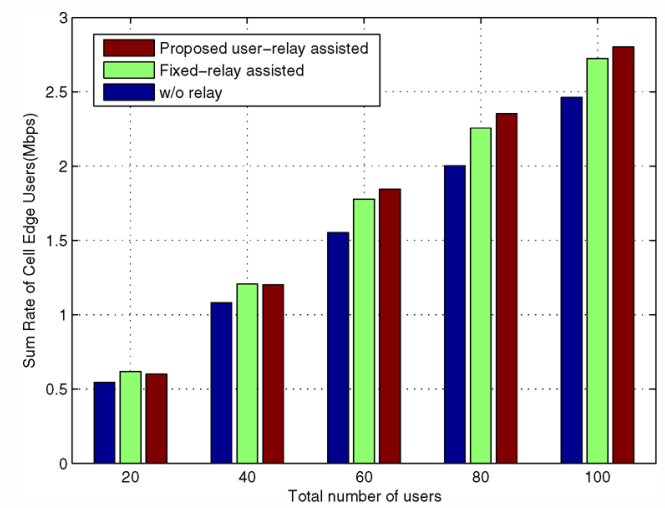

Fig. 3: Sum rate of cell edge users for MISO-OFDMA.

The MISO-OFDMA based schemes are compared in terms of percentage of satisfied users as given in Figure 2. As expected, the $w / o$ relay scheme has the lowest percentage of satisfied users, since relay assisted schemes have a chance to communicate via a relay when the direct link is not good enough to communicate. The proposed user-relay assisted scheme also outperforms the fixed-relay assisted scheme over 
60 users because not only the number of users but also the number of user-relays is increasing. It is obvious that the users' density is important for the relaying opportunity in the userrelay assisted scheme.

In Figure 3, the cell-edge users' data rate as a function of total number of users is illustrated since we let only cell-edge users to use relays for the user-relay and fixed relay assisted schemes in order to increase the data rate of these users. Not only fixed relays but also user-relays increase the cell-edge users' data rates compared to w/o relay scheme according to this figure. The simulation results show an increase in the celledge capacity ranging from $11 \%$ to $15 \%$ by using fixed relays and $10 \%$ to $19 \%$ by using user-relays depending on the number of users so the cell-edge users are not victimized because of their locations to the BS. Moreover, the proposed user-relay assisted scheme improved the cell-edge users' data rate $4 \%$ on average compared to the fixed relay assisted scheme.

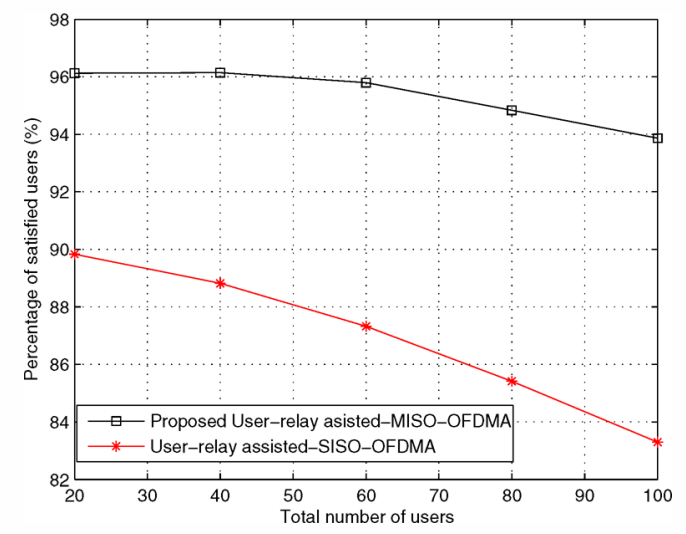

Fig. 4: Percentage of satisfied users-MISO vs SISO OFDMA.

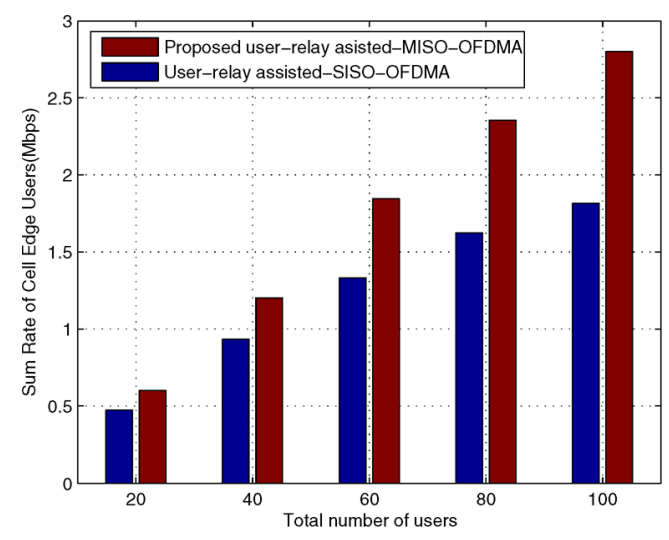

Fig. 5: Sum rate of cell-edge users-MISO vs SISO OFDMA.

Finally, the proposed user-relay assisted MISO-OFDMA scheme is compared to the user-relay assisted SISO-OFDMA scheme in order to examine the effect of multiple antennas on OFDMA based relaying technology. The percentage of satisfied users and the cell-edge users' data rates are compared in Figures 4 and 5 respectively. It can be seen that the MISOOFDMA case in which more than one link is supported on each subchannel has higher percentage of satisfied users and celledge users' data rate than SISO-OFDMA case. The satisfied users' percentage is increased between $7 \%$ and $12 \%$ and the cell-edge users' data rate is improved ranging from $27 \%$ to $53 \%$.

\section{CONCLUSION}

In this paper, we have developed sub-optimum RA algorithm for the MISO-OFDMA based user-relay assisted networks. The proposed RA algorithm revealed the advantages of using multiple antennas and user-relaying together. The numerical results showed us that the proposed user-relay assisted MISO-OFDMA scheme increased the percentage of satisfied users and data rate of the cell-edge users when compared to existing non-relaying and fixed relay assisted MISO-OFDMA schemes and user-relay assisted SISO-OFDMA scheme.

\section{REFERENCES}

[1] H. Yanikomeroglu, "Fixed and mobile relaying technologies for cellular networks" in Second Workshop on Applications and Services in Wireless Networks (ASWN), pp.75-81, Paris, July 2002.

[2] R. Pabst et al., "Relay-Based Deployment Concepts for Wireless and Mobile Broadband Radio" IEEE Communications Magazine, vol. 42(9), pp.80-89, 2004.

[3] C. Hoymann et al., "Relaying Operation in 3GPP LTE: Challenges and Solutions," IEEE Communications Magazine, vol. 50(2), pp.156-162, 2012.

[4] M. Tehrani, M. Uysal, H. Yanikomeroglu, "Device-to-device communication in 5G cellular networks: challenges, solutions, and future directions" IEEE Communications Magazine, vol. 52(5), pp.86-92, 2014

[5] WH. Chin, Z. Fan, R. Haines, "Emerging technologies and research challenges for $5 \mathrm{G}$ wireless networks", IEEE Wireless Communications, vol. 21(2), pp.106-112, 2014.

[6] Z. Han, T. Himsoon, WP Siriwongpairat, KJR Liu, "Resource allocation for multiuser cooperative OFDM networks: who helps whom and how to cooperate". IEEE Transactions on Vehicular Technology, vol. 58(5), pp.2378-2391, 2009.

[7] W. Shim, Y. Han, S. Kim, "Fairness-aware resource allocation in a cooperative OFDMA uplink system", IEEE Transactions on Vehicular Technology, vol. 59(2), pp.932-939, 2010.

[8] TCY. Ng, W. Yu, "Joint optimization of relay strategies and resource allocations in cooperative cellular networks", IEEE Journal on Selected Areas in Communications, vol. 25(2), pp.328-339, 2007.

[9] L. Weng, RD. Murch, "Cooperation strategies and resource allocations in multiuser OFDMA systems". IEEE Transactions on Vehicular Technology, vol. 58(5), pp.2331-2342, 2009.

[10] L. Shenghong, RD. Murch, "Realizing Cooperative Multiuser OFDMA Systems with Subcarrier Resource Allocation", IEEE Transactions on Wireless Communications, vol. 12(4), pp.1923-1935, 2013.

[11] I. Basturk, B. Ozbek, C. Edemen, AS. Tan, E. Zeydan, S. Ergut, "Radio Resource Management for OFDMA-Based Mobile Relay Enhanced Heterogenous Cellular Networks", in Proc. IEEE Vehicular Technology Conference (VTC)-Spring, pp.1-5, Dresden, June 2013.

[12] I. Basturk, B. Ozbek, D. Le Ruyet, "Queue Aware Resource Allocation for OFDMA-Based Mobile Relay Enhanced Networks", in International Symposium on Wireless Communication Systems (ISWCS), pp.1-5, Ilmenau, August 2013.

[13] V. Sreng, H. Yanikomeroglu, DD. Falconer, "Relay selection strategies in cellular networks with peer-to-peer relaying", in Proc. IEEE Vehicular Technology Conference (VTC)-Fall, pp.1949-1953, Orlando, FL, 2003.

[14] Z.Tu, RS. Blum, "Multiuser diversity for a dirty paper approach", IEEE Communications Letters, vol. 7(8), pp.370-372, 2003. 